\title{
A REMARK ON EXTENSION OF ORDER PRESERVING OPERATOR INEQUALITY
}

\section{TAtsuya Koizumi and Keilchi Watanabe}

Abstract. We will give an extension of order preserving operator inequality of Furuta type.

Mathematics subject classification (2010): 47A63.

Keywords and phrases: Löwner-Heinz inequality, Furuta inequality, order preserving operator inequality.

\section{REFERENCES}

[1] T. ANDo AND F. HiAI, Log majorization and complementary Golden-Thompson type inequalities, Linear Algebra Appl. 197/198 (1994), 113-131.

[2] T. FURUTA, $A \geqslant B \geqslant 0$ assures $\left(B^{r} A^{p} B^{r}\right)^{1 / q} \geqslant B^{(p+2 r) / q}$ for $r \geqslant 0, p \geqslant 0, q \geqslant 1$ with $(1+2 r) q \geqslant$ $p+2 r$, Proc. Amer. Math. Soc. 101, 1 (1987), 85-88.

[3] T. FURUTA, Extension of the Furuta inequality and Ando-Hiai log-majorization, Linear Algebra Appl. 219 (1995), 139-155.

[4] T. FURUTA, Invitation to linear operators, Taylor \& Francis, London, 2001.

[5] T. FurutA, A proof of an order preserving inequality, Proc. Japan Acad. Ser. A Math. Sci. 78, 2 (2002), 26.

[6] T. FURUTA, Further extension of an order preserving operator inequality, J. Math. Inequal. 2, 4 (2008), 465-472.

[7] T. FURUTA, Log majorization via an order preserving operator inequality, Linear Algebra Appl. 431, 1-2 (2009), 132-138.

[8] T. FURUTA, Operator function associated with an order preserving operator inequality, J. Math. Inequal. 3, 1 (2009), 21-29.

[9] T. FURUTA, An extension of order preserving operator inequality, Math. Inequal. Appl. 13, 1 (2010), 49-56.

[10] E. HEInZ, Beiträge zur Störungstheorie der Spektralzerlegung, Math. Ann. 124 (1951), 415-438.

[11] K. LöWNER, Über monotone Matrixfunktionen, Math. Z. 38 (1934), 177-216.

[12] M. UchiYama, Criteria for monotonicity of operator means, J. Math. Soc. Japan 55, 1 (2003), 197207.

[13] C. YANG AND Y. WANG, Further extension of Furuta inequality, J. Math. Inequal. 4, 3 (2010), $391-$ 398. 